EPJ Web of Conferences 41, 04027 (2013)

DOI: $10.1051 /$ epjconf/20134104027

(C) Owned by the authors, published by EDP Sciences, 2013

\title{
Probing the origin of fluorescence quenching of a graphene-porphyrin hybrid material
}

\author{
Divya Sharma $^{1, a}$, Xiaoyan Zhang $^{2}$, Ben L. Feringa ${ }^{2}$, Wesley R. Browne ${ }^{2}$, Jennifer L. Herek ${ }^{1}$, and \\ Annemarie Huijser ${ }^{1}$ \\ 1 Optical Sciences group, MESA+ Institute for Nanotechnology, University of Twente, PO Box 217, \\ 7500AE Enschede, The Netherlands \\ 2 Stratingh Institute for Chemistry, University of Groningen, Nijenborgh 4, 9747AG Groningen, The \\ Netherlands
}

\begin{abstract}
We report transient absorption spectroscopic studies on the hybrid material composed of porphyrin molecules covalently attached to graphene for investigating the mechanism underlying the reported fluorescence quenching of porphyrin in the hybrid [1]. Excited state dynamics of pure graphene suspension and porphyrin have also been studied as reference samples. A fast excited state decay was observed in the hybrid.
\end{abstract}

\section{Introduction}

Graphene, a two-dimensional single atomic layer of carbon atoms, has been a topic of intense scientific research due to its unusual electronic band structure which leads to interesting physical phenomena, e.g., anomalous quantum hall effect [2], spin-resolved quantum interference [3] and bipolar supercurrent [4]. In addition, graphene-based hybrid nanomaterials, which combine the unique optical, electrical, magnetic and chemical properties of each component have the potential applications in solar cells, sensors and catalysis. Such a hybrid material combining graphene with porphyrin, namely grapheneTPP (tetraphenylporphyrin), has been recently synthesized by cycloaddition reactions [1]. Further, fluorescence spectroscopic studies [1] on graphene-TPP and free TPP-CHO (5-(4-Formylphenyl)10,15,20-triphenylporphyrin) in dimethylformamide (DMF) (containing similar concentration of porphyrin) have shown that while TPP-CHO shows strong fluorescence $\left(\lambda_{\text {peak }}: 650 \mathrm{~nm}\right.$ and $\left.710 \mathrm{~nm}\right)$, fluorescence of porphyrin is quenched significantly in graphene-TPP. The reduced fluorescence lifetime $(<500 p s)$ of the graphene-TPP as compared to that of free TPP-CHO $(\sim 10 \mathrm{~ns})$ further confirms the fast quenching of the porphyrin singlet excited state in the hybrid material. In addition, the fluorescence quantum yield is reduced from $4 \%$ in TPP-CHO to $0.3 \%$ for the graphene-TPP hybrid. This has been attributed to either an energy or electron transfer process from the covalently attached porphyrin molecules to graphene. However, an ultrafast time-resolved spectroscopic study which can resolve the mode of interaction and the excited state dynamics of graphene and porphyrin in the hybrid material is still lacking. It is to be noted that such transient studies can also provide insight into the nature of the photoproduct resulting from the $S_{1}$ state quenching. To this end, we have conducted femtosecond transient pump-probe spectroscopy studies on the graphene-TPP hybrid.

\section{Experimental details}

A $420 \mathrm{~nm}$ pump beam (corresponding to the blue side excitation of the Soret band of porphyrin centered at $419 \mathrm{~nm}, S_{0}$ to $S_{2}$ state) is obtained by frequency doubling the nearinfrared output (800-900

a e-mail: d.sharma@utwente.nl

This is an Open Access article distributed under the terms of the Creative Commons Attribution License 2.0, which permits unrestricted use, distribution, and reproduction in any medium, provided the original work is properly cited. 

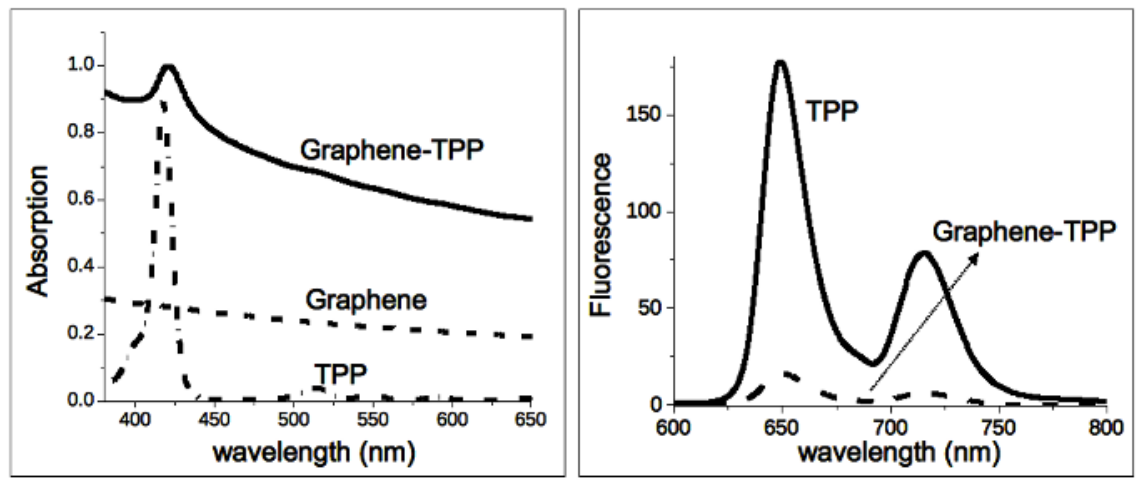

Fig. 1. (a) Absorption spectra of TPP-CHO (dash-dotted line), graphene (dashed line) and graphene-TPP (solid line) in DMF, (b) Fluorescence spectra of TPP-CHO (solid line) and graphene-TPP (dashed line).
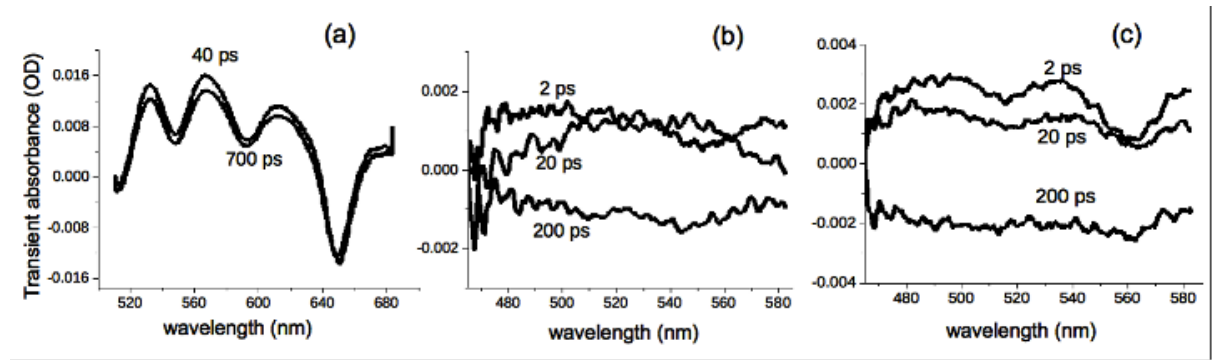

Fig. 2. Transient absorption spectra as a function of time delays for (a) porphyrin (b) graphene and (c) grapheneporphyrin hybrid in DMF

nm) obtained from the Noncollinear Optical Parametric Amplifier (NOPA) in a BBO crystal. The NOPA was pumped by the second harmonic $(387 \mathrm{~nm})$ of the fundamental $775 \mathrm{~nm}, 150 \mathrm{fs}$ pulses from a commercial $1 \mathrm{kHz}$ regeneratively amplified mode-locked Ti:Sapphire laser system (CPA-2001, Clark-MXR Inc.). A white light continuum (covering the spectral range from $465 \mathrm{~nm}$ to $685 \mathrm{~nm}$ ), generated by focusing the fundamental in a $2 \mathrm{~mm}$ Sapphire crystal, is used as the probe. The polarization of pump and probe beams are parallel. The experimental time resolution is $\sim 300 \mathrm{fs}$.

\section{Results and Discussion}

Femtosecond pump-probe time-resolved absorption spectroscopic studies on the graphene-TPP hybrid in DMF were performed with a pump excitation at $420 \mathrm{~nm}$. Excited state dynamics of free TPP-CHO and pure graphene suspension in DMF were also studied, as reference samples.

Figure 1 (a) shows the steady-state absorption spectra of graphene-TPP hybrid, free TPP-CHO and graphene in DMF. Figure 1(b) shows the fluorescence spectra of the hybrid and free TPP-CHO in DMF. Figure 2(a-c) displays the transient absorption spectra of free TPP-CHO, graphene and the hybrid as a function of pump-probe delay times. For free TPP-CHO (Fig. 2a) ground state bleaching ( $S_{0}$ to $S_{1}$ transition) is clearly observed as a dip in the transient absorption spectra at $515 \mathrm{~nm}$ and additional dips at $550 \mathrm{~nm}, 590 \mathrm{~nm}$ and $646 \mathrm{~nm}$ can be assigned to a combination of ground state bleaching (GSB), excited state absorption (ESA) and stimulated emission (centered at $650 \mathrm{~nm}$ ). The transient spectra of the hybrid (Fig. 2c) shows an ESA at early delays $(<30 p s)$ which was observed to decay rapidly. The dips at $520 \mathrm{~nm}$ and $560 \mathrm{~nm}$ clearly indicate the signature of the porphyrin in the hybrid. On the other hand, pure graphene suspension shows a broad ESA which decays gradually over time. 


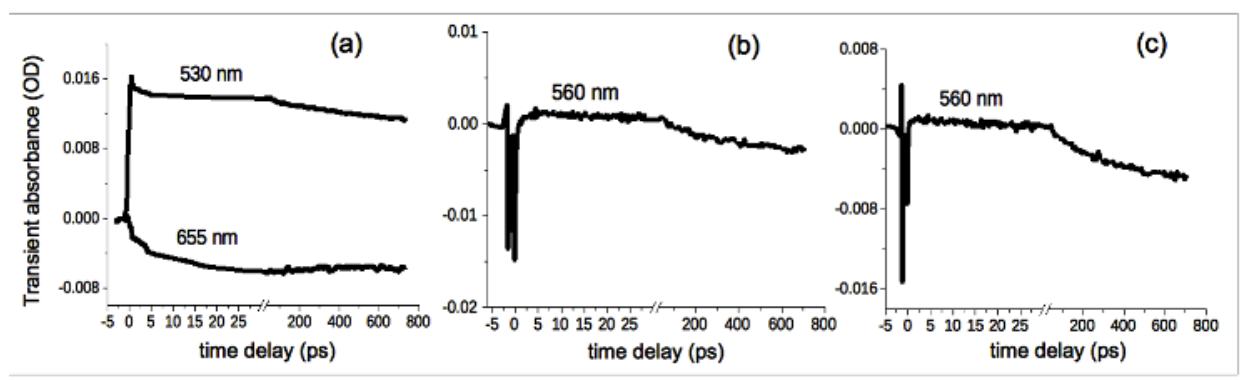

Fig. 3. Kinetic traces as a function of wavelength for (a) porphyrin (b) graphene and (c) graphene -porphyrin hybrid in DMF.

Figure 3(a-c) presents the temporal evolution of the differential absorption signals of TPP-CHO graphene and the hybrid. In TPP-CHO, the kinetic trace (Fig. 3(a)) at the blue wavelength (530 nm) exhibits a positive absorption immediately following the photoexcitation which can be assigned to ESA from the $S_{1}$ state. A subsequent initial fast relaxation followed by a slower decrease is observed. On the other hand, the kinetics at the red wavelength $(655 \mathrm{~nm})$ shows a negative absorption indicating the evolution of the stimulated emission band. The ingrowth of this band can be attributed to the internal conversion from the $S_{2}$ to the $S_{1}$ state occurring on the picosecond timescale. The kinetic trace of graphene (Fig. 3(b)) at $560 \mathrm{~nm}$ shows that the signal exhibits a sharp decrease within the 1 ps timescale following photoexcitation. This initial drop can be attributed to the photogeneration of carriers by the pump pulse thereby resulting in increased transmissivity of the probe. This is followed by a sharp positive rise which exhibits an initial fast relaxation over a few ps time scales. This can be attributed to the carrier-carrier intraband scattering [5] which equilibrates the photogenerated carriers among themselves and with the intrinsic charge carriers. The signal then shows a long lived negative transient signal which does not decay till 700 ps. The graphene-TPP hybrid shows a similar rapid rise of ESA followed by a fast decay.

Exponential decay fits to the singlet excited state decay of porphyrin in the hybrid reveal a double exponential decay with an initial ultrafast decay of $\sim 7 \mathrm{ps}$, followed by a slower decay of $\sim 0.2 \mathrm{~ns}$. The ultrafast timescale can be attributed to the rapid quenching of the singlet excited state of porphyrin. Pure graphene suspension shows only a longer single exponential decay $(\sim 0.2 n s)$. To note, the accurate determination of this longer timescale $(0.2 \mathrm{~ns})$ is out of reach of the $0.8 \mathrm{~ns}$ scan range of the present experiment.

Experiments are currently underway to increase the signal-to-noise ratio to investigate the formation of porphyrin radical cation species $(>600 \mathrm{~nm})$. We are also exploring the possibility of increasing the loading of porphyrin.

\section{References}

1. X. Zhang, L. Hou, A. Cnossen, A. C. Coleman, O. Ivashenko, P. Rudolf, B. J. van Wees, W. R. Browne and B. L. Feringa, Chem. Eur. J. 17, (2011) 8957.

2. K. S. Novoselov, A. K. Geim, S. V. Morozov, D. Jiang, M. I. Katsnelson, I. V. Grigorieva, S. V. Dubonos and A. A. Firsov, Nature 438, (2005) 197.

3. M. B. Lundeberg and J. A. Folk, Nat. Phys. 5, (2009) 894.

4. H. B. Heersche, P. J. Herrero, J. B. Oostinga, L. M. K. Vandersypen and A. F. Morpurgo, Nature 446, (2007) 56.

5. J. M. Dawlaty, S. Shivaraman, M. Chandrashekhar, F. Rana and M.G. Spencer, Appl. Phys. Lett. 92, (2008) 042116. 